\title{
Multiple-speech-source localization using advanced histogram mapping method
}

\author{
Yusuke Hioka $^{1}$, Masao Matsuo ${ }^{2}$ and Nozomu Hamada ${ }^{2, *}$ \\ ${ }^{1}$ NTT Cyber Space Laboratories, NTT Corporation, \\ 3-9-11 Midori-cho, Musashino, 180-8585 Japan \\ ${ }^{2}$ Signal Processing Lab., School of Integrated Design Engineering, Keio University, \\ 3-14-1 Hiyoshi, Kouhoku-ku, Yokohama, 223-8522 Japan
}

(Received 18 June 2008, Accepted for publication 21 August 2008)

Keywords: DOA estimation, Microphone array, Multiple-sound-source localization, Histogram mapping method

PACS number: 43.60.Jn [doi:10.1250/ast.30.143]

\section{Introduction}

Speech source localization is used for a number of applications, such as tele-conference systems and hands-free automatic voice recognition systems. The most basic approach to finding the direction of a speech source is to estimate the time difference of arrival (TDA) between the sensors of a microphone array. Knapp and Carter [1] introduced the generalized cross-correlation (GCC) method in which the TDA is estimated from a weighted crosscorrelation of the input signals. There have been several modifications of the GCC method that are effective in estimating the direction of a single source, but they are not applicable for simultaneously active multiple sources.

A long-standing method for multiple-sound-source localization is the delay-and-sum beamformer method [2]. However, the resolution capability of this method is limited, which makes it impractical for use in some applications. Wang and Kaveh [3] proposed coherent signal-subspace (CSS) processing for multiple wide-band signal source localization. This method is based on signal-subspace processing [4], which was originally developed for narrow-band direction of arrival (DOA) estimation. The simulation results presented in [3] demonstrate the high resolution of CSS processing. Regardless of this advantage, the method requires preliminary DOA estimation, and the accuracy of the final DOA estimate is strongly dependent on the preliminary estimation. In addition, this method involves a high computational cost due to the eigenvalue analysis of the covariance matrix with respect to the array input signal.

In this study, we propose a method for estimating DOAs of multiple speech signals based on a histogram mapping (HM) method [5]. The HM method estimates TDAs for each microphone pair in a triangular array. However, in contrast to the GCC, it estimates TDAs independently from each frequency bin of the input signal, then it derives the final DOA estimate as the average of the histograms of the estimated TDAs. The HM method has several advantages over other conventional methods. 1) We can estimate the DOAs of multiple speech signals, even if the number of active speech sources exceeds the number of microphone elements. 2) It requires no preliminary estimation of DOAs. 3) The estima-

\footnotetext{
*e-mail: hamada@sd.keio.ac.jp
}

tion has a low computational cost. Despite these merits, its DOA estimation accuracy remains a problem to be solved. The estimation of the TDA at a microphone pair degrades when the direction of the speech source deviates from the broadside direction [6,7]. Because the estimation accuracy at one microphone pair differs from that at the other two pairs due to the triangular configuration, taking the average of the DOA estimates obtained from these different pairs is not appropriate. To resolve this problem, in the proposed method we introduce a delay vector and a coordinate transformation based on it. This avoids the average DOA calculation for the three pairs and improves the estimation accuracy.

\section{Histogram mapping method}

The HM method is applied to the triangular microphone array shown in Fig. 1. In this configuration, there are three microphones located at the vertices of an equilateral triangle whose inter microphone distance is $d$, and they receive speech signals $s_{k}(n) ; k=1,2, \ldots, K$ propagating from the direction $\theta_{k}$. Here, $K$ represents the number of speech sources.

The input signal at each microphone is first decomposed into its time-frequency components by a short-time Fourier transform (STFT). When multiple speakers are simultaneously active (i.e. $K \geq 2$ ), each sensor receives a mixture of multiple speech signals. Generally, for most spectra, different speech signals rarely overlap in the time-frequency domain. This is called the $\mathrm{W}$-disjoint orthogonality (W-DO) property [8]: a single-frequency bin of the input signal at any frame contains the spectral component of only one speech signal. Consequently, by decomposing the input signal into its timefrequency components by the STFT transform, the multiplesource localization problem is transformed into a set of narrow-band, single-source problems.

As a consequence of W-DO, the transformed input signal at the $i$ th microphone $(i=1,2,3)$ is modelled as follows:

$$
X_{i}(m, \omega)=S(m, \omega) e^{-j \omega \tau_{i}} .
$$

Here, $m$ and $\omega$ represent the frame number and the angular frequency, respectively. $S(m, \omega)$ is the STFT of the dominant speech signal at an arbitrary cell $(m, \omega)$, and $\tau_{i}$ represents the signal delay at the $i$ th microphone with respect to the reference point, usually taken to be the centre of the array. 


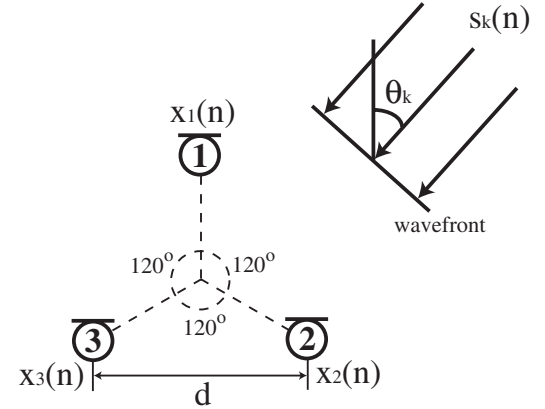

Fig. 1 Triangular microphone array.

The TDAs are estimated from the phase angle differences between a microphone pair, $i$ and $j(i \neq j)$ [9].

$$
\tau_{i j}(m, \omega)=\frac{1}{\omega} L \frac{X_{i}(m, \omega)}{X_{j}(m, \omega)}
$$

Then $\tau_{i j}(m, \omega)$ is transformed to the DOA $\theta_{i j}(m, \omega)$ by the following relations:

$$
\begin{aligned}
& \theta_{12}(m, \omega)=\arcsin \left(\frac{c}{d} \tau_{12}(m, \omega)\right)-\frac{2}{3} \pi \\
& \theta_{23}(m, \omega)=\arcsin \left(\frac{c}{d} \tau_{23}(m, \omega)\right) \\
& \theta_{31}(m, \omega)=\arcsin \left(\frac{c}{d} \tau_{31}(m, \omega)\right)+\frac{2}{3} \pi,
\end{aligned}
$$

where $c$ represents the speed of sound. Each $\theta_{i j}(m, \omega)$ is mapped into a histogram, and then the three histograms are averaged and smoothed in order to remove insignificant small peaks. The number of peaks in the resultant histogram is equal to the number of active speakers, and the centre of each peak is the estimated DOA of a signal from an active speaker.

\section{Proposed method}

Let us consider a plane wave travelling from the direction angle $\theta$. We define a TDA vector, which consists of the TDAs of the three microphone pairs and is given by

$$
\tau(\theta)=\left[\tau_{12}(\theta), \tau_{23}(\theta), \tau_{31}(\theta)\right]^{\mathrm{T}},
$$

where the theoretical values of the TDAs are given by

$$
\begin{aligned}
\tau_{12}(\theta) & =\frac{d}{c} \sin \left(\theta+\frac{2}{3} \pi\right) \\
\tau_{23}(\theta) & =\frac{d}{c} \sin (\theta) \\
\tau_{31}(\theta) & =\frac{d}{c} \sin \left(\theta-\frac{2}{3} \pi\right) .
\end{aligned}
$$

The linear equation $\tau_{12}(\theta)+\tau_{23}(\theta)+\tau_{31}(\theta)=0$ is satisfied, and thesquared norm of $\tau(\theta)$ takes a constant value of $\tau_{12}^{2}(\theta)+\tau_{23}^{2}(\theta)+\tau_{31}^{2}(\theta)=3 d^{2} / 2 c^{2}$. This means that the locus of $\tau(\theta)$ is a circle whose radius is $\sqrt{3 / 2} d / c$ as $\theta$ varies from $-\pi$ to $\pi$. Thus, the azimuth $\theta$ is estimated by projecting the three-dimentional (3D) coordinates of $\tau(\theta)$ so that the new $x y$ plane contains the projected circular locus of $\tau(\theta)$ as shown in Fig. 2. The projection matrix $\boldsymbol{T}$ is defined as

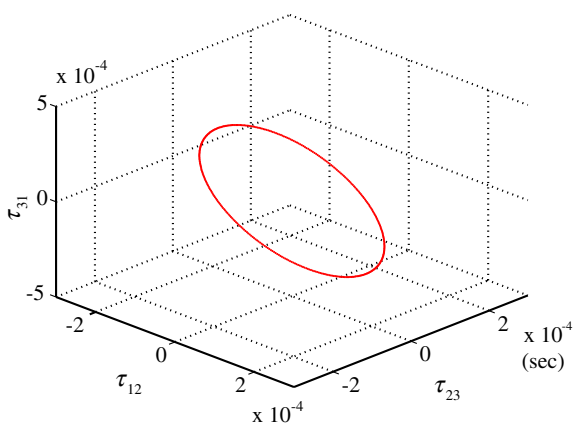

Fig. 2 Locus of $\tau(\theta)$.

$$
\boldsymbol{T}=\left[\boldsymbol{e}_{1}, \boldsymbol{e}_{2}, \boldsymbol{e}_{3}\right]
$$

where the column vectors $\boldsymbol{e}_{1}, \boldsymbol{e}_{2}$, and $\boldsymbol{e}_{3}$ are defined to be orthonormal.

$$
\begin{aligned}
\boldsymbol{e}_{1} & \triangleq \frac{1}{\|\tau(\pi / 2)\|} \tau(\pi / 2) \\
& =\left[-\sqrt{\frac{1}{6}}, \sqrt{\frac{2}{3}},-\sqrt{\frac{1}{6}}\right]^{\mathrm{T}} \\
\boldsymbol{e}_{2} & \triangleq \frac{1}{\|\tau(0)\|} \tau(0) \\
& =\left[\sqrt{\frac{1}{2}}, 0,-\sqrt{\frac{1}{2}}\right]^{\mathrm{T}} \\
\boldsymbol{e}_{3} & =\left[\sqrt{\frac{1}{3}}, \sqrt{\frac{1}{3}}, \sqrt{\frac{1}{3}}\right]^{\mathrm{T}}
\end{aligned}
$$

The new coordinate system $[x, y, z]$ is derived by

$$
\begin{aligned}
\tau^{\prime \mathrm{T}} & =\boldsymbol{T}^{\mathrm{T}} \tau(\theta) \\
& =\left[\begin{array}{lll}
\sqrt{\frac{3}{2}} \frac{d}{c} \sin \theta & \sqrt{\frac{3}{2}} \frac{d}{c} \cos \theta & 0
\end{array}\right]^{\mathrm{T}} .
\end{aligned}
$$

From Eq. (14), the DOA $\theta$ is obtained after determining the $x$ and $y$ components of the transformed vector $\tau^{\prime}(\theta)$ by the following equation:

$$
\theta=\arctan \frac{x}{y}
$$

In accordance with these theoretical relationships, we implement the estimation algorithm using the observed signals. The TDA vector at each time-frequency cell is obtained by integrating the estimated TDAs as

$$
\tau(m, \omega)=\left[\tau_{12}(m, \omega), \tau_{23}(m, \omega), \tau_{31}(m, \omega)\right]^{\mathrm{T}} .
$$

Then the azimuth estimate at each cell is obtained by

$$
\theta(m, \omega)=\arctan \frac{x(m, \omega)}{y(m, \omega)},
$$

where $x(m, \omega)$ and $y(m, \omega)$ are the components of the vector $\tau^{\prime}(m, \omega)$ defined by

$$
\begin{aligned}
\tau^{\prime}(m, \omega) & :=\boldsymbol{T}^{\mathrm{T}} \tau(m, \omega) \\
& =[x(m, \omega), y(m, \omega), z(m, \omega)]^{\mathrm{T}} .
\end{aligned}
$$


Here the frequency band is limited to $\omega<\pi c / d$ in order to avoid the spatial aliasing effect. Finally, we form a histogram of the estimated $\theta(m, \omega)$ obtained at each time-frequency cell. The peaks correspond to the directions of the arrival azimuth angles.

In practice, the sound sources may not always be located on the array plane. Thus, we have to take into consideration the elevation angle $\phi$, which is defined to have the array plane of $\phi=0$, of the speech source location. Consequently, Eqs. (7)-(9) can be replaced by the following equations:

$$
\begin{aligned}
\tau_{12}(\theta, \phi) & =\frac{d}{c} \cos \phi \sin \left(\theta+\frac{2}{3} \pi\right) \\
\tau_{23}(\theta, \phi) & =\frac{d}{c} \cos \phi \sin (\theta) \\
\tau_{31}(\theta, \phi) & =\frac{d}{c} \cos \phi \sin \left(\theta-\frac{2}{3} \pi\right) .
\end{aligned}
$$

Compared with Eqs. (7)-(9), every component of the TDA vector is multiplied by $\cos \phi$. Hence, Eq. (14) is rewritten as

$$
\begin{aligned}
\tau^{\prime}(\theta, \phi) & =\boldsymbol{T}^{\mathrm{T}} \tau(\theta) \cos \phi \\
& =\left[\begin{array}{lll}
\sqrt{\frac{3}{2}} \frac{d}{c} \cos \phi \sin \theta & \sqrt{\frac{3}{2}} \frac{d}{c} \cos \phi \cos \theta & 0
\end{array}\right]^{\mathrm{T}} .
\end{aligned}
$$

Note from Eq. (22) that the radius $r$ of the circle is multiplied by $\cos \phi$. Regardless of this variation, Eq. (15) is valid for estimating the azimuth angle exactly. Therefore, the proposed DOA estimation procedure is also applicable to speech sources even if they do not lie on the array plane (except for the cases of $\phi=\pi / 2$ and $-\pi / 2$ ).

\section{Performance evaluation}

\subsection{Results of computer simulation}

The array input signals used in the computer simulation are generated by delaying dry speech signals [10] with appropriate samples depending on the source direction $\theta$ [11], and a Gaussian white noise is added to simulate the sensor noise. The simulation parameters are listed in Table 1 . The frame length was determined in order to set the bandwidth of each frequency bin to be $10-20 \mathrm{~Hz}$, which is the most appropriate bandwidth for analyzing a speech signal [12].

4.1.1. Evaluation of resolution

First, we evaluate the resolution of the estimation compared with the beamforming method [2]. Figure 3 shows the estimated DOAs when two different speech sources are simultaneously active. One speaker is set at $0^{\circ}$, and the direction of the other speaker is varied from $10^{\circ}$ to $180^{\circ}$ at $10^{\circ}$ intervals. The horizontal axis corresponds to the true direction

Table 1 Simulation parameters.

\begin{tabular}{cc}
\hline Input SNR & $14 \mathrm{~dB}$ \\
Sampling frequency & $16,000 \mathrm{~Hz}$ \\
Wave velocity $c$ & $340 \mathrm{~m} / \mathrm{s}$ \\
Microphone distance $d$ & $0.08 \mathrm{~m}$ \\
Window & Hamming \\
STFT point & 1,024 \\
Frame length & 1,024 samples \\
Frame overlap & 512 samples \\
Length of snapshot & 4,096 samples $(256 \mathrm{~ms})$ \\
\hline
\end{tabular}

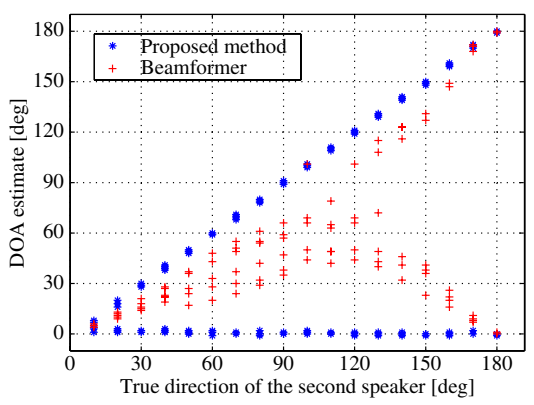

Fig. 3 DOA estimation for two active sources.



Fig. 4 RMSE of DOA estimation for multiple active sources.

of the latter speaker, and the vertical axis denotes the estimated DOA for both speakers.

As can be observed from the figure, the proposed method is able to estimate the DOA for both speakers with very low deviation errors. The directions of the two speakers are correctly determined even when their directions are only $20^{\circ}$ apart. In contrast, the beamformer method gives estimation results with a significant amount of bias and deviation errors. 4.1.2. Estimation accuracy for multiple active sources

Next, we compare the estimation accuracy of the proposed method with the original HM method. Figure 4 shows the root-mean-square error (RMSE) of the estimated DOAs when multiple speakers are active. The horizontal axis represents the number of active sources.

The simulation is conducted as follows. When evaluating the performance for two active speech sources for example, two speaker locations are randomly selected from directions that are multiples of $45^{\circ}$, i.e., $0,45, \cdots, 315$. For each pair of source locations, DOA estimation is performed 50 times, and the RMSE defined by Eq. (23) is calculated.

$$
R M S E=\sqrt{\sum_{n=1}^{N}\left(\bar{\theta}_{n}-\theta\right)^{2} / N}
$$

Here, $\theta$ represents the true direction, $\bar{\theta}_{n}$ represents the estimated direction in the $n$-th trial, and $N$ represents the total number of estimates. The same procedure is conducted for 40 randomly selected pairs of source locations, and the RMSEs calculated for the 40 pairs are averaged to give the final RMSE. Similarly, the evaluations for three/four active speeches are conducted by randomly choosing three/four directions.

From Fig. 4, the RMSE of the proposed method is $51 \%$ of that of the original HM method when two speakers are active, 


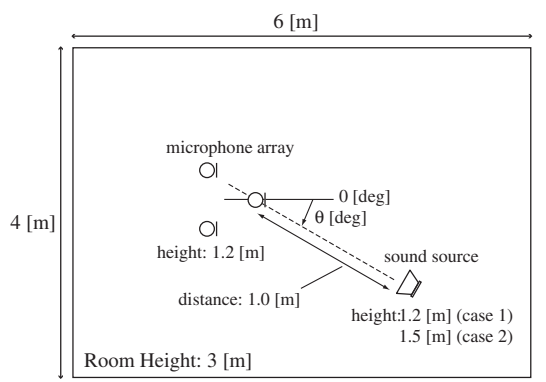

Fig. 5 Experimental setup.

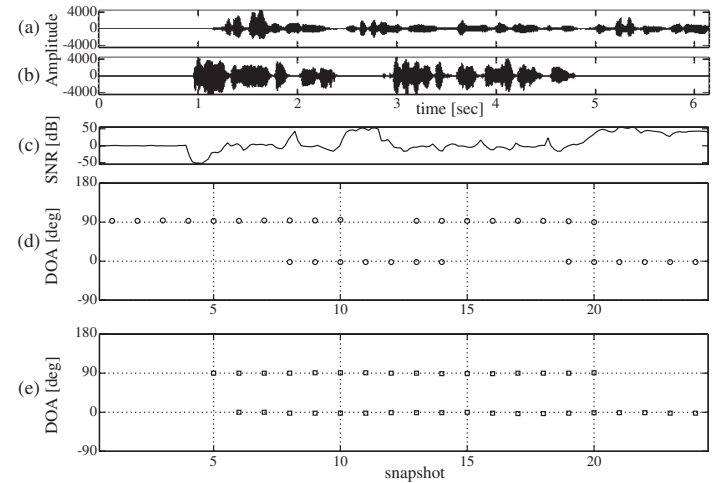

Fig. 6 DOA estimation for two active sources in a real acoustic environment: (a) waveform of signal 1 $\left(\theta_{1}=0^{\circ}\right)$, (b) waveform of signal $2\left(\theta_{2}=90^{\circ}\right)$, (c) segmental SNR (signal 2 is recognized as noise), (d) DOAs estimated by the HM method, (e) DOAs estimated by the proposed method.

$57 \%$ when three speakers are active, and $67 \%$ when four speakers are active.

\subsection{Experimental results}

To verify the effectiveness of the proposed method in a real acoustic environment, we performed an experiment in a reverberant room. As illustrated in Fig. 5, two sources are positioned $1 \mathrm{~m}$ away from the centre of the microphone array in the directions of $\theta_{1}=0^{\circ}$ and $\theta_{2}=90^{\circ}$. The source signals are human voices speaking Japanese sentences of about $6 \mathrm{~s}$ in length, and they speak almost simultaneously. We evaluated the proposed method for two different heights of the source from the floor, $1.2 \mathrm{~m}$ (case 1: $\phi=0^{\circ}$ ) and $1.5 \mathrm{~m}$ (case 2: $\phi \neq 0^{\circ}$ ). Figures 6(d) and 6(e) show the DOA estimation results for the HM method and the proposed method, respectively. Note that the HM method occasionally fails to detect the direction of signal 1 as the segmental signal-tonoise ratio (SNR) shown in Fig. 6(c) decreases. The segmental SNR in this case is calculated by recognizing signal 1 as the "signal" and signal 2 as the "noise."

As shown in Fig. 7, the estimation accuracy of the proposed method compared with the HM method is also confirmed in a real acoustic environment. Furthermore, the proposed method gives accurate results both for case 1 and case 2 . This fact demonstrates the applicability of the proposed method even if the source position slightly deviates from the array plane.

\section{Conclusion}

In this study, we proposed a method for improving the

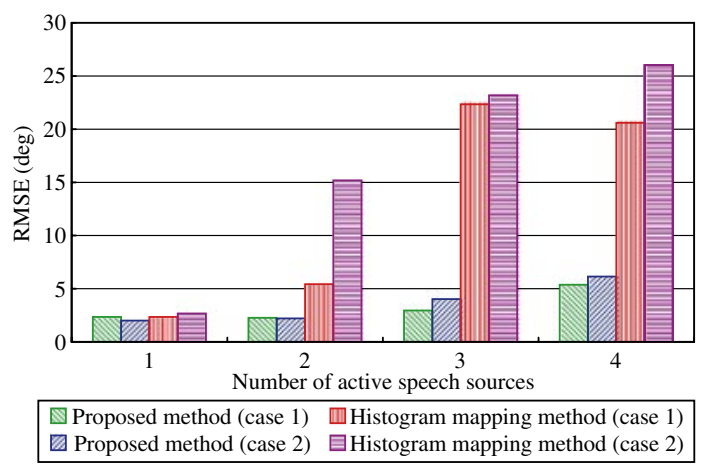

Fig. 7 RMSE of estimated DOAs in real acoustic environment.

performance of the HM method. In the proposed method, the three estimated TDAs are integrated into a vector form and are collectively used for DOA estimation. Through computer simulation and an actual experiment, we confirmed that the proposed method outperforms the original HM method in both estimating DOA and detecting the correct number of speech sources.

\section{References}

[1] C. H. Knapp and G. Carter, "The generalized correlation method for estimation of time delay," IEEE Trans. Acoust. Speech Signal Process., 24, 320-327 (1976).

[2] N. Kikuma, "Beamformer method," in Adaptive Signal Processing with Array Antenna (Science and Technology Publishing Company, Inc., Tokyo, 1999), pp. 178-181 (in Japanese).

[3] H. Wang and M. Kaveh, "Coherent signal-subspace processing for the detection and estimation of angles of arrival of multiple wide-band sources," IEEE Trans. Acoust. Speech Signal Process., 33, 823-831 (1985).

[4] R. O. Schmidt, "Multiple emitter location and signal parameter estimation," IEEE Trans. Antennas Propag., 34, 276-280 (1986).

[5] J. Huang, N. Ohnishi and N. Sugie, "A biomimetic system for localization and separation of multiple sound sources," IEEE Trans. Instrum. Meas., 44, 733-738 (1995).

[6] Y. Hioka and N. Hamada, "DOA estimation of speech signal using microphones located at vertices of equilateral triangle," IEICE Trans. Fundam., E87-A, 559-566 (2004).

[7] Y. Hioka and N. Hamada, "Tracking of speaker direction by integrated use of microphone pairs in equilateral-triangle," IEICE Trans. Fundam., E88-A, 633-641 (2005).

[8] O. Yilmaz and S. Rickard, "Blind separation of speech mixtures via time-frequency masking," IEEE Trans. Signal Process., 52, 1830-1847 (2004).

[9] A. G. Piersol, "Time delay estimation using phase data," IEEE Trans. Acoust. Speech Signal Process., 29, 471-477 (1981).

[10] S. Itahashi, "Continuous speech corpus for research Vol. 1-3," Japan Information Processing Development Center (Edited by the Acoustical Society of Japan) (1991).

[11] J. Ohga, Y. Yamazaki and Y. Kaneda, Acoustic Systems and Digital Processing for Them (IEICE, Tokyo, 1995), pp. 215216 (in Japanese).

[12] M. Aoki, M. Okamoto, S. Aoki, H. Matsui, T. Sakurai and Y. Kaneda, "Sound source segregation based on estimating incident angle of each frequency component of input signals acquired by multiple microphones," Acoust. Sci. \& Tech., 22, 149-157 (2001). 